\title{
Vasculitis masquerading as aortic valve endocarditis
}

\section{B Iqbal, N G Fisher, K M Fox}

Heart 2005;91:e37 (http://www.heartjnl.com/cgi/content/full/91/5/e37). doi: 10.1136/hrt.2005.060525

\begin{abstract}
Small vessel vasculitis and endocarditis can both present with multisystem involvement and may present a diagnostic dilemma. Renal and cardiac involvement is common in small vessel vasculitis and rarely small vessel vasculitis may cause heart block. When a patient presents with diffuse symptoms, deteriorating renal function, and heart block, endocarditis and vasculitis should be included in the differential diagnosis. The case is discussed of a man with a history of aortic valve endocarditis who presented again with similar symptoms, deteriorating renal function, and heart block. There was no evidence of aortic valve endocarditis with abscess formation. A renal biopsy confirmed small vessel vasculitis and the patient responded promptly to immunosuppressive treatment. Correct diagnosis is essential in such cases, as immunosuppression in true endocarditis can be catastrophic. In this case, with the correct diagnosis, immunosuppression proved life saving and prevented erroneous aortic valve surgery.
\end{abstract}

$\mathrm{V}$ asculitides comprise a spectrum of diseases with multisystem involvement and are classified as small, medium, or large vessel disease. Small vessel vasculitis includes Wegener's granulomatosis, microscopic polyarteritis, and Churg-Strauss syndrome, often affecting the kidneys and causing a rapidly progressive glomerulonephritis with crescent formation. Cardiac involvement is also recognised in small vessel vasculitis. Endocarditis may also present in a similar fashion with multisystem involvement posing a diagnostic challenge. We present an extraordinary case of a patient with a history of aortic valve endocarditis who presented again in a similar fashion with heart block and deteriorating renal function. He was found to have diffuse small vessel vasculitis.

\section{CASE HISTORY}

A 54 year old man presented with fever, scleritis, arthralgia, a vasculitic rash, and severe colitis. A full autoimmune screen was negative. Inflammatory bowel disease was diagnosed and treatment with steroid was begun. He subsequently developed a toxic megacolon and underwent a total colectomy. Histopathological examination of the colectomy specimen confirmed ulcerative colitis. His symptoms persisted and blood cultures yielded methicillin resistant Staphylococcus aureus. Transoesophageal echocardiography (TOE) showed vegetations on the aortic valve. Aortic valve endocarditis was diagnosed and the patient was treated successfully with vancomycin, rifampicin, and meropenem for six weeks. A follow up TOE showed no vegetations.

He presented again, nine months after the initial presentation, with a one week history of fever, worsening scleritis, widespread arthralgia, and a vasculitic rash. On examination he had a temperature of $38.5^{\circ} \mathrm{C}$, pulse rate was 70 beats $/ \mathrm{min}$ (irregular), and blood pressure was 79/18 $\mathrm{mm} \mathrm{Hg}$. The jugulovenous pressure was not raised. Cardiovascular examination showed an early diastolic murmur, and the chest was clear to auscultation. Abdominal examination was unremarkable and there was no evidence of hepatosplenomegaly. The remainder of the examination was unremarkable.

The initial laboratory investigations showed haemoglobin $10.1 \mathrm{~g} / \mathrm{dl}$, white cell count $12.5 \times 10^{9} / \mathrm{l}$, platelets $492 \times 10^{9} / \mathrm{l}$, prothrombin time 14.1 seconds, activated partial thromboplastin time 32.2 seconds, sodium $135 \mathrm{mmol} / \mathrm{l}$, potassium $5.8 \mathrm{mmol} / \mathrm{l}$, urea nitrogen $12.9 \mathrm{mmol} / \mathrm{l}$, creatinine $306 \mu \mathrm{mol} / \mathrm{l}$, bilirubin $10 \mu \mathrm{mol} / \mathrm{l}$, alkaline phosphatase $94 \mathrm{U} / \mathrm{l}$, alanine aminotransferase $14 \mathrm{U} / \mathrm{l}$, albumin $21 \mathrm{~g} / \mathrm{l}$, and $\mathrm{C}$ reactive protein $259 \mathrm{mg} / \mathrm{l}$. Urinalysis was strongly positive for blood and protein. A chest radiograph was unremarkable and a 12 lead ECG confirmed atrioventricular block. Recurrence of aortic valve endocarditis was suspected and empirical treatment with teicoplanin, gentamicin, and rifampicin was begun. TOE showed moderate aortic regurgitation with no evidence for an aortic root abscess. Cardiac magnetic resonance imaging confirmed the absence of an aortic root abscess. He continued to have swinging pyrexias and repeated blood cultures were negative. His renal function continued to deteriorate and an ultrasound scan of the kidneys was unremarkable. Repeated ECGs showed persisting atrioventricular block (fig 1 ).

The persistence of symptoms with antibiotic treatment and rising inflammatory markers led us to suspect an alternative diagnosis of vasculitis. A full autoimmune screen was negative. Serum complement and immunoglobulins were within normal limits. In view of worsening renal function (urea nitrogen $16.4 \mathrm{mmol} / \mathrm{l}$ and creatinine $479 \mu \mathrm{mol} / \mathrm{l}$ ), renal support with haemofiltration was begun. A renal biopsy was carried out to define the underlying pathology. Light microscopy showed focal and segmental necrotising glomerulonephritis with crescent formation being consistent with a small vessel vasculitis (fig 2). Immunofluorescence studies showed no glomerular localisation of immunoglobulin or complement. Pauci-immune glomerulonephritis with systemic involvement was diagnosed. Antibiotic treatment was stopped and immunosuppression was promptly started. The patient responded well to treatment, and within five days heart block resolved completely.

\section{DISCUSSION}

This case highlights how systemic manifestations of endocarditis can mimic those of small vessel vasculitis. A compelling feature in this case was the recent history of aortic valve endocarditis with similar presentation, which initially favoured the diagnosis of recurrent aortic valve endocarditis. Heart block is a rare complication of ulcerative colitis, which may be another cause of heart block given the patient's history of ulcerative colitis. However, this cause is unlikely in our patient, who has had a total colectomy, as heart block in ulcerative colitis usually occurs in the setting of active colitis.

The heart is often involved in a spectrum of vasculitic diseases. In rheumatoid arthritis, rheumatoid granulomas

Abbreviations: $\mathrm{TOE}$, transoesophageal echocardiography 


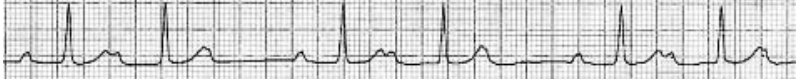

Figure 1 ECG strip showing atrioventricular block.

may involve the myocardium, endocardium, and valves. Arteritis affecting small vessels may produce pericarditis and rarely cardiac ischaemia. ${ }^{2}$ Conduction disturbances are rare, though heart block has been reported in rheumatoid arthritis. ${ }^{3}$ Polyarteritis nodosa is a medium-large vessel vasculitis, with cardiac involvement in up to $80 \%$ of cases. These typically manifest as pericarditis, arrhythmias, coronary arteritis, and myocardial infarction. ${ }^{4}$ Similarly, cardiac involvement is seen in up to $70 \%$ of cases of systemic lupus erythematosus. The most common findings are pericarditis, myocarditis, and Libman-Sacks endocarditis. ${ }^{5}$ Congenital heart block is a characteristic feature of neonatal lupus syndrome, which is associated with transplacental transfer of anti-Ro and anti-La antibodies. Arrhythmias are uncommon but heart block has been reported in systemic lupus erythematosus. ${ }^{6}$

Small vessel vasculitides include Wegener's granulomatosis, microscopic polyarteritis, and Churg-Strauss syndrome. They are usually associated with the presence of antineutrophil cytoplasmic antibodies, though this may be negative. ${ }^{7}$ Wegener's granulomatosis is a necrotising granulomatous vasculitis with predilection for the upper and lower respiratory tract and the kidneys. Cardiac involvement has been reported in $8-44 \%$ of cases. This typically manifests as pericarditis, coronary arteritis, and myocarditis. Other manifestations are endocarditis or valvulitis, conduction system granulomas, arteritis of the atrioventricular nodal artery, myocardial infarction, and epicarditis. ${ }^{8}$ Complete heart block, although rare, has been reported in Wegener's granulomatosis. ${ }^{9}$

Small vessel vasculitis affects the kidney causing a rapidly progressive glomerulonephritis with few immune deposits, hence termed "pauci-immune glomerulonephritis". ${ }^{7}$ This may test negative for antineutrophil cytoplasmic antibodies, as seen in the present case. In our patient, renal biopsy confirmed a diagnosis of pauci-immune glomerulonephritis with diffuse systemic involvement. Arteritis of the atrioventricular nodal artery would account for the heart block observed in this patient. The complete resolution of heart block with immunosuppressive treatment provided compelling evidence to support the diagnosis. This case illustrates how the clinical features of vasculitis may mimic aortic valve endocarditis. When a patient presents with atrioventricular conduction block and diffuse systemic symptoms, small vessel vasculitis should be included in the differential diagnosis. Correct diagnosis of such cases is important, as appropriate immunosuppression may prove to be life saving.

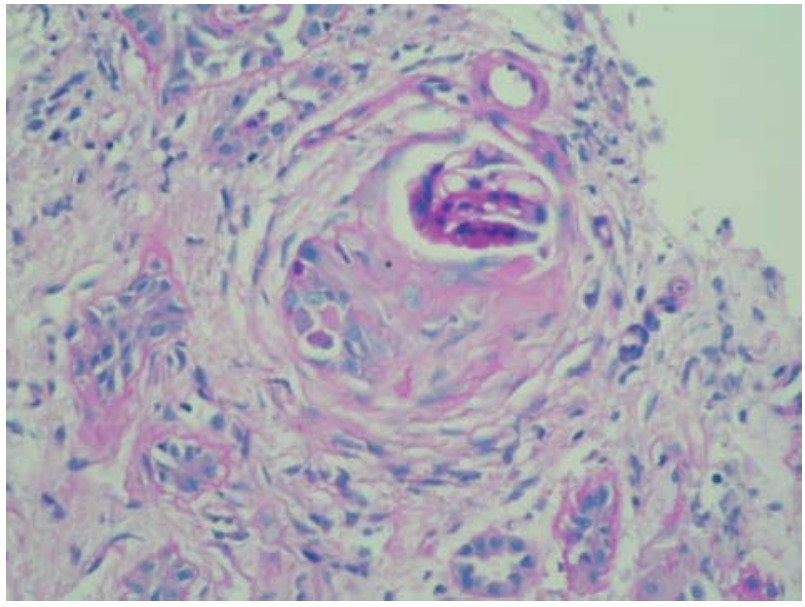

Figure 2 Light microscopy with periodic acid-Schiff (PAS) stain showing a glomerulus with a fibrocellular crescent.

\section{ACKNOWLEDGEMENTS}

We thank Dr T Cook, St Mary's Hospital, London for providing the histopathological image and reviewing the discussion of the case.

\section{Authors' affiliations \\ M B Iqbal, N G Fisher, K M Fox, Department of Cardiology, Royal Brompton Hospital, London, UK}

Correspondence to: Dr M Bilal labal, Department of Cardiology, Level 3, Royal Brompton Hospital, Sydney Street, London SW3 6NP, UK; biqbal@excite.com

Accepted 25 January 2005

\section{REFERENCES}

1 Maeder HU. The complete heart-block: an extraintestinal manifestation of ulcerative colitis. Z Gastroenterol 1996;34:27-9.

2 Nomeir AM, Turner R, Watts E, et al. Cardiac involvement in rheumatoid arthritis. Ann Intern Med 1973;79:800-6.

3 Adhiyaman V, Oke A, Aziz K, et al. Progressive heart block in active rheumatoid arthritis. Int J Clin Pract 2000;54:544-5.

4 James TN, Birk RE. Pathology of the cardiac conduction system in polyarteritis nodosa. Arch Intern Med 1966;1 17:561-7.

5 Roberts WC, High ST. The heart in systemic lupus erythematosus. Curr Probl Cardiol 1999;24:1-56.

6 Gomez-Barrado JJ, Garcia-Rubira JC, Polo Ostariz MA, et al. Complete atrioventricular block in a woman with systemic lupus erythematosus. Int J Cardiol 2002;82:289-92

7 Hedger N, Stevens J, Drey N, et al. Incidence and outcome of pauci-immune rapidly progressive glomerulonephritis in Wessex, UK: a 10-year retrospective study. Nephrol Dial Transplant 2000;15:1593-9.

8 Grant SCD, Levy RD, Venning MC, et al. Wegener's granulomatosis and the heart. Br Heart J 1994;71:82-6.

9 Forstot JZ, Overlie PA, Neufeld GK, et al. Cardiac complications of Wegener granulomatosis: a case report of complete heart block and review of the literature. Semin Arthritis Rheum 1980;10:148-54. 